DOI

\title{
СОВЕРШЕНСТВОВАНИЕ ДИАГНОСТИКИ И ЛЕЧЕНИЯ РАЗЛИЧНЫХ МОРФОЛОГИЧЕСКИХ ФОРМ ЭХИНОКОККОЗА ПЕЧЕНИ
}

\author{
๑А. М. Шамсиев, Ш. А. Юсупов, З. Б. Курбаниязов, К. Э. Рахманов \\ Самаркандский государственный медицинский институт, Республика Узбекистан
}

РЕЗЮМЕ. Цель работы: поиск путей оптимизации диагностических и лечебных мероприятий у больных с эхинококкозом печени с учётом морфологических форм паразитарных кист.

Материалы исследования. Исследование основано на изучении результатов применения различных вариантов оперативных вмешательств при лечении 305 больных с эхинококкозом печени. Все пациенты за последние 10 лет (2005-2015) прошли лечение во II клинике Самаркандского государственного медицинского института.

Результаты исследования. Послеоперационные осложнения были отмечены у 35 (11,5 \%) больных, послеоперационная летальность отмечена в 1 (0,3 \%) случае. Рецидив эхинококкоза имел место у 17 из 236 наблюдавшихся больных в отдаленном периоде $(77,4 \%)$.

Выводы: Результаты хирургического лечения эхинококковых кист печени во многом зависят от дифференцированного подхода к выбору адекватного способа оперативного лечения, учета морфологической формы эхинококковой кисты и соблюдения принципов апаразитарности и антипаразитарности. В отдаленном периоде рецидив эхинококкоза наблюдали у 2,9 \% больных, оперированных по поводу осложненного эхинококкоза, а также у 4,2 \% больных, отказавшихся от химиотерапии в послеоперационном периоде.

КЛЮчЕВЫЕ СЛОВА: эхинококкоз печени, морфологические формы, диагностика, лечение.

Введение. Эхинококкоз - распространенное паразитарное заболевание, встречающееся по всему миру, которое чаще всего поражает печень и легкие (от 44 до 88 \%), реже наблюдается поражение других органов [2, 5-7, 9].

Изучение морфологических модификаций эхинококка человека описаны в работахЖ.А. Шамсиева (2015). Автором были изучены в основном морфология зародышевых элементов эхинококка (лавроцист, выводковых капсул и протосколексов) и морфологические изменения, происходящие в пораженных эхинококкозом органах человека [7].

Исследователи отмечают, что при эхинококковой болезни возникают и прогрессируют ряд нарушений клеточного и гуморального иммунитета. При этом существует мнение, что способность возбудителя подавлять или искажать иммунный ответ хозяина-носителя следует относить к факторам патогенности, т.к. степень тяжести формирующегося иммунодефицитного состояния коррелирует с его вирулентностью $[1,2,4,6,8]$.

В свете изложенного актуальным представляется уточнение вопросов, касающихся морфологических модификаций эхинококкоза. Какая форма существования паразита наиболее агрессивна и приводит к множественным формам поражения органов? При какой морфологической модификации следует более всего опасаться рецидива заболевания? Влияют ли эти морфологические формы на хирургическую тактику? Эти вопросы послужили поводом для нашей исследовательской работы.

Цель работы: поиск путей оптимизации диагностических и лечебных мероприятий у боль- ных эхинококкозом печени с учётом морфологических форм паразитарных кист.

Материал и методы исследования. Исследование основано на изучении результатов применения различных вариантов оперативных вмешательств при лечении 305 больных с эхинококкозом печени. Все пациенты за последние 10 лет (2005-2015) прошли лечение во II клинике Самаркандского государственного медицинского института.

Исследование носило как ретроспективный, так и проспективный характер. Наблюдения охватывали больных с завершенным хирургическим лечением, у которых были удалены до- и интраоперационно диагностированные кисты печени.

Первичный эхинококкоз имелся у 279 (91,5 \%) больных, вторичный (рецидивный) - у 26 (8,5 \%). Одиночные кисты печени выявлены у 227 (74,4 \%) больных, множественные - у 78 (25,6 \%). При монокистозном эхинококкозе размеры кист составили от 4,0 до 25,0 см в диаметре, а при множественном эхинококкозе печени - от 3,0 до 15,0 см. Среди больных с солитарными кистами печени правая доля была поражена в 180 (59,0 \%) наблюдениях, левая - в 64 (21,0%). Эхинококкоз центральной локализации отмечен в 61 (20,0%) наблюдении.

Осложненный эхинококкоз встречался у 84 $(27,5 \%)$ пациентов, из них нагноение эхинококковой кисты - в 37 случаях, прорыв в свободную брюшную полость - в 8 случаях, прорыв в билиарный тракт - в 9, прорыв в плевральную полость и далее в бронхиальное дерево - в 1 случае. Частичное или полное обызвествление фиброзной 
Огляди літератури, оригінальні дослідження, погляд на проблему

капсулы имелось у 13 пациентов, разрушение фиброзной капсулы с открытием в её просвет желчных свищей - у 16.

Проведено морфологическое исследование хитиновых оболочек эхинококковых кист печени, удаленных во время оперативного вмешательства, для изучения их структуры в зависимости от стадии жизнедеятельности паразита. Нами установлено, что echinococcus veterenorum имелся у $150(49,2 \%)$ больных, echinococcus hominis - y 109 $(35,7 \%)$ больных, echinococcus acephalocysticus у 35 (11,5\%) больных и ассоциация echinococcus hominis + echinococcus veterinorum была отмечена у 11 (3,6 \%) пациентов (рис. 1).
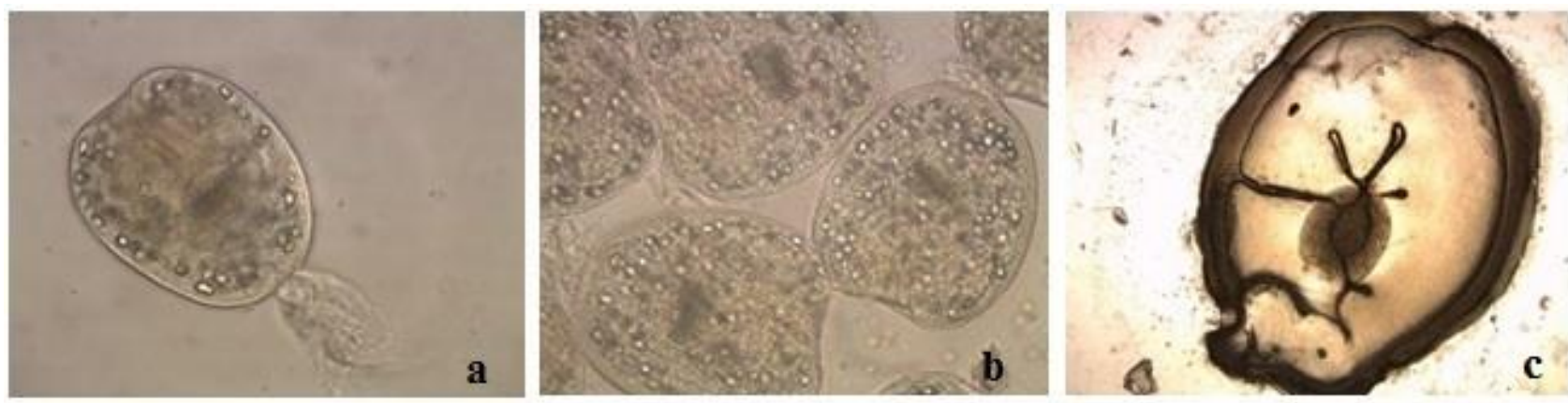

Рис. 1. Схема строения лавроцист эхинококка различных морфологических модификаций: a - echinococcus veterinorum, $b$ - echinococcus hominis, $c$ - echinococcus acephalocystis.

Для определения морфологических модификаций эхинококкоза мы провели анализ результатов инструментальных методов исследования (УЗИ, КТ) и изучили операционный материал.

Прежде чем дать характеристику морфологической структуры модификаций эхинококкоза необходимо отметить, что все они отличались друг от друга в первую очередь наличием и степенью выраженности дистрофических процессов, протекающих в их оболочках, особенно в герминативной оболочке, где имеется основная структурная единица паразита - выводковая капсула, способная продуцировать жизнеспособные протосколексы [7].

Модификация echinococcus hominis отличается тем, что внутри кисты, помимо гидатидной жидкости, выводковых капсул с протосколексами имеются дочерние, а иногда и внучатые пузыри.

Обычно такие кисты бывают больших и средних размеров. Материнские кисты макроскопически имеют матовую шероховатую поверхность и окрашены в молочно-белый или беловатожелтый цвет. Число дочерних цист колеблется в широких пределах - от одной до нескольких десятков. Обычно при нагноении или отмирании материнской кисты такие же изменения претерпевают находящиеся в их просвете дочерние. Однако в больших эхинококковых кистах дочерние пузыри погибают в разные сроки, в связи с чем, наряду с мертвыми, могут обнаруживаться и живые дочерние пузыри [7].

Давление гидатидной жидкости в этих кистах обычно низкое, а цвет жидкости прозрачный или чаще мутный. При цитологическом исследовании жидкости материнской кисты и зрелых дочерних кист обнаруживаются протосколексы.

При световой микроскопии на герминативной оболочке обнаружены выводковые капсулы в виде выпячиваний продолговатой формы величиной от 0,015 до 0,045мм. Дистрофические изменения носят очаговый характер. Различные участки стенки кисты могут отличатся друг от друга по выраженности изменений. Набухание и расслоение зародышевой оболочки свидетельствует о нарушении проницаемости оболочек. В умерших кистах отмечается ранняя гибель герминативного слоя. Хитиновая оболочка более устойчивая и распаду подвергается позже.

Дооперационная диагностика модификации echinococcus hominis возможна при помощи инструментальных методов исследования УЗИ и КТ.

В дооперационном периоде модификация echinococcus hominis на УзИ была определена у 81 больного. Характерным ультразвуковым признаком при этой форме паразита являлся симптом «рыбьей чешуи», которая бывает двух видовв виде уплощенных и округлых чешуек. Первые являются признаками «многокамерного» эхинококка, а вторые - симптомами инволюции кисты. Чувствительность УЗИ по выявлению модификации еchinococcus hominis по нашим данным составила $74,3 \%$. УзИ семиотика echinococcus hominis представлена на рисунке 2 [7].

Обзорная рентгенография брюшной полости не имеет специфических признаков, характерных для морфологических форм эхинококкоза печени. Она применялась у всех пациентов. Высокое 
Огляди літератури, оригінальні дослідження, погляд на проблему

стояние купола диафрагмы справа обнаружено

симптом характерен для локализации кисты на у 40 (13,1 \%) больных. Этот рентгенологический

диафрагмальной поверхности печени.

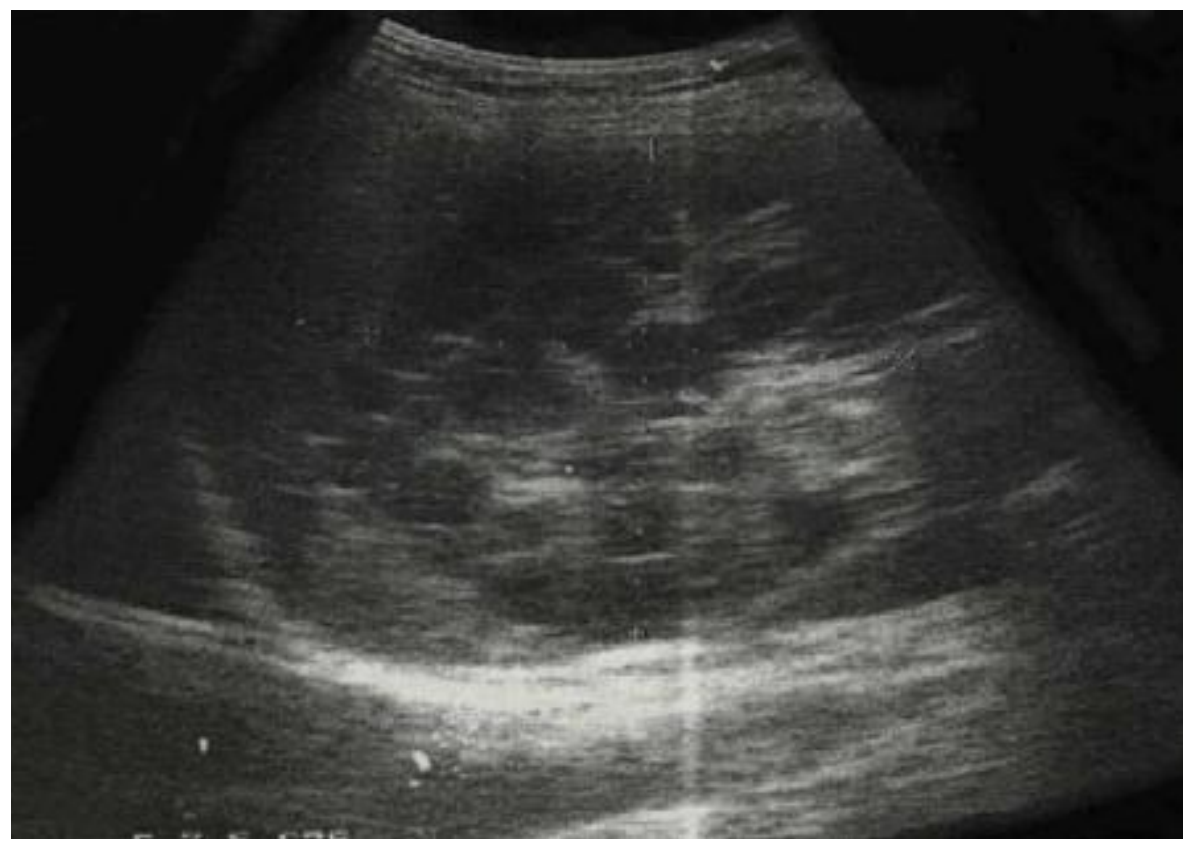

Рис. 2. УзИ картина дочерних пузырей.

В 6 (1,9\%) случаях при обзорной рентгеноскопии брюшной полости выявлены обызвествленные кисты. Таким образом, по нашим данным информативность рентгенологического исследования при эхинококкозе печени составила 15,1\%.

Клиническое значение выявления echinococcus hominis заключается в том, что, являясь стадией ранних посмертных изменений именно при этой форме отмечается миграция сколексов за пределы хитиновой оболочки в толщу, или даже за пределы фиброзной капсулы и происходит экзогенное почкование при росте эхинококковой кисты.

Проведенный нами анализ результатов хирургического лечения эхинококкоза в отдаленном послеоперационном периоде является свидетельством того, что именно при этой модификации наблюдались рецидивы заболевания на месте ранее существовавших кист. Так, из 17 (5,6 \%) рецидивов болезни 10 (3,3\%) приходилось на случаи заражения echinococcus hominis.

Поэтому, по нашему мнению, одним из способов профилактики рецидивов эхинококкоза печени является полноценная и адекватная санация остаточной полости после эхинококкэктомии именно при наличии дочерних пузырей в материнской полости. Наиболее эффективным в этом плане является использование 30 \% раствора хлорида натрия и 100 \% раствора глицерина [7].

Модификация echinococcus veterinorum отмечена у 150 (49,2 \%) пациентов. При этой форме заболевания внутри лавроцист имеются только выводковые капсулы и эхинококковая жидкость. Образование дочерних пузырей не происходит. Особенностью этого вида пузырей является наибольшее, по сравнению с другими формами, давление эхинококковой жидкости. В большинстве наблюдений такие кисты клинически описываются как «напряженные».

При цитологическом исследовании эхинококковой жидкости в ней обнаруживаются множество сколексов и выводковых капсул, свободно плавающих в эхинококковой жидкости. Их количество в 1 мл жидкости в среднем составляет 1200, микроскопически сколексы имеют овальную форму. При световой микроскопии внутренний зародышевый слой тонкий, в виде мембраны выстилает изнутри полость пузыря. Набухание и расслоение этого слоя менее выражены. Почти вся поверхность герминативной оболочки покрыта сплошным слоем зародышевых пузырей, которые придают герминативной оболочке зернистость, наподобие песчинки.

Echinococcus veterinorum является, пожалуй, самой агрессивной формой существования паразита, что обусловлено высоким давлением гидатидной жидкости, содержащей большое число жизнеспособных сколексов, которые при малейшем нарушении целостности оболочки попадают в свободную брюшную полость, вызывая массивное поражение органов брюшной полости эхинококкозом. Также характерным признаком данно- 
Огляди літератури, оригінальні дослідження, погляд на проблему

го вида кист является толстая фиброзная капсула, формирующаяся вокруг эхинококковой кисты. Поэтому оперативные вмешательства при этой модификации должны выполнятся с более тщательным соблюдением правил апаразитарности и антипаразитарности. Точная дооперационная диагностика кист этой модификации несколько затруднена, что объясняется отсутствием специфической симптоматики. Однако есть ряд косвен- ных признаков, на основании которых можно с большей долей вероятности судить о наличии echinococcus veterinorum. Прежде всего, это «гидатидный песок», который выявляется при УЗИ при изменении положении тела исследуемого. Такая эхографическая картина вызывается множеством сколексов, заполняющих полость материнской кисты и проявляющихся в виде гиперэхогенного осадка (рис. 3).

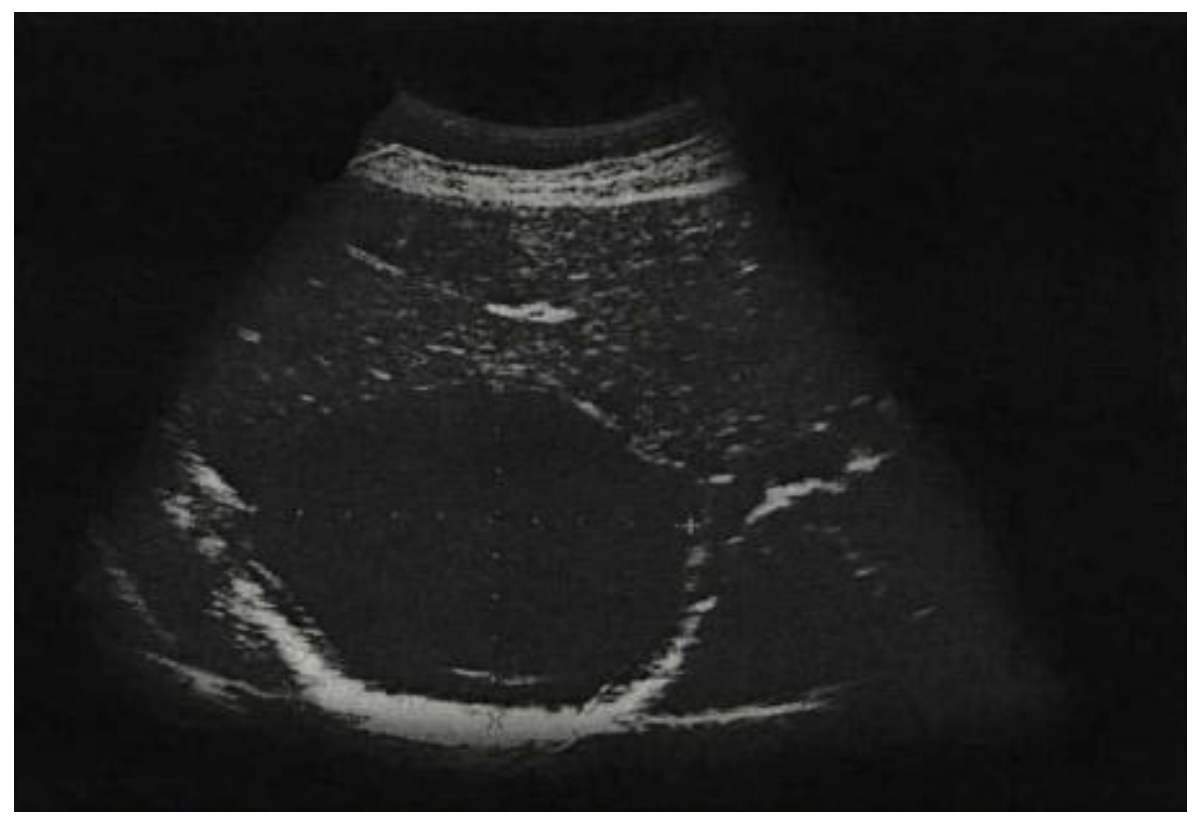

Рис. 3. УзИ печени. Эхинококковая киста с наличием гиперэхогенного осадка.

Еще одним из отличительных признаков, способных дать косвенное представление о модификации кисты, является толщина фиброзной капсулы. По нашим данным, косвенные признаки echinococcus veterinorum до операции определены у 29 (9,5 \%) пациентов.

Лавроцисты третьей модификации echinococcus acephalocystis были отмечены в 35 (11,5 \%) наблюдениях. Данные виды кист характеризуются отсутствием выводковых капсул и протосколексов и чаще встречаются у животных, чем у человека. Они обычно бывают средних размеров, диаметром не более 6-7 см, имеют желтоватосерую окраску, отличаются ослизненной стенкой, фиброзная капсула не столь выражена.

При цитологическом исследовании гидатидной жидкости кист сколексы не обнаружены. Гистологическое исследование герминативной оболочки с помощью светового микроскопа показало, что вся ее поверхность подвергается дистрофическим изменениям, выводковые капсулы отсутствуют. Поэтому эти кисты не способны продуцировать зародышевые элементы.

Дооперационная диагностика этой модификации кист представляет большие трудности. Эхо- графическая и компьютерно-томографическая характеристики таких кист имеют большое сходство с непаразитными кистами печени, так как содержимое их однородное и толщина фиброзной капсулы не достигает больших размеров. Именно поэтому достаточно часто возникает необходимость в проведении дифференциальной диагностики данных видов кист с непаразитарными кистами печени. Нами с помощью УЗИ в 17 (5,6 \%) случаях дооперационно установлены косвенные признаки модификации еchinococcus acephalocystis.

Нами выяснено, что echinococcus acephalocystis является наименее инвазивной формой существования паразита. «Благоприятность» течения обусловлена тем, что, имея невысокую степень агрессии за счет отсутствия жизнеспособных элементов в эхинококковой жидкости и благодаря низкому энергетическому потенциалу, при данной модификации кист не наблюдается случаев диссеминированного эхинококкоза и рецидивов заболевания. Кроме того, данные виды кист не достигают больших размеров и морфологические изменения со стороны печени носят обратимый характер. 
Огляди літератури, оригінальні дослідження, погляд на проблему

Клиническое значение дооперационного выявления echinococcus acephalocystis заключается в том, что при этой модификации целесообразно применение малоинвазивных методов лечения, таких как чрескожно-пункционная методика лечения и применение лапараскопических операций.

При множественном эхинококковом поражении печени, которое констатировано у 78 (25,6 \%) больных, в некоторых случаях наблюдалось сочетание различных модификаций эхинококкоза. Преимущественно наблюдалась ассоциация echinococcus hominis + echinococcus veterinorum, что было отмечено у 11 (3,6 \%) пациентов.

Каждая из указанных морфологических модификаций эхинококкоза печени характеризуется специфичностью развития, инвазивностью, а также различным прогнозом результатов хирургического лечения и реабилитации. Знание особенностей и дооперационная диагностика морфологических модификаций эхинококкоза печени дают клиницисту информацию, которая оказывает существенное значение при планировании оперативного вмешательства и дальнейшей реабилитации пациентов.

Однако возможности дооперационного определения морфологичеких модификаций эхинококкоза печени в плане инструментальной и лабораторной диагностики до настоящего времени ограничены, что диктует необходимость совершенствования данных методов.

При изучении отдаленных результатов спустя 3, 6 месяцев и 1 год после радикального оперативного вмешательства основное внимание было сосредоточено на тех сведениях, которые позволяют оценить тактику хирургического лечения рецидивного эхинококкоза печени. Среди них мы учитывали следующие данные:

1) наличие или отсутствие жалоб;

2) результаты дополнительных методов исследования;

3) выявление рецидивных эхинококковых кист в печени и легком.

К рецидивам эхинококкоза относили случаи возникновения новых эхинококковых кист через 12 месяцев после радикального хирургического вмешательства.

Неудовлетворительные результаты имели место у 17 из 236 наблюдавшихся больных в отдаленном периоде (77,4 \%). У 7 больных рецидив эхинококкоза в легких и эхинококкоз печени в отдаленном послеоперационном периоде был обусловлен диссеминацией эхинококкового процесса, у больных, оперированных по поводу осложненного эхинококкоза, прорыва в брюшную полость и желчные протоки. У 10 больных рецидив эхинококкоза наблюдали у отказавшихся от химиотерапии в послеоперационном периоде (табл. 1).

Таблица 1. Результаты хирургического лечения эхинококкоза печени в отдаленном послеоперационном периоде

\begin{tabular}{|c|c|c|c|c|c|c|}
\hline \multirow{3}{*}{$\begin{array}{c}\text { Рецидив в зависимости } \\
\text { от морфологических } \\
\text { модификаций }\end{array}$} & \multirow{3}{*}{$\begin{array}{l}\text { Количество } \\
\text { наблюдений в } \\
\text { отд. периоде }\end{array}$} & \multirow{2}{*}{\multicolumn{2}{|c|}{$\begin{array}{c}\text { К-во } \\
\text { рецидивов }\end{array}$}} & \multicolumn{3}{|c|}{ Причина рецидива } \\
\hline & & & & \multirow{2}{*}{$\begin{array}{c}\text { операция } \\
\text { по поводу } \\
\text { прорыва } \\
\text { кисты в } \\
\text { брюшную } \\
\text { полость }\end{array}$} & \multirow{2}{*}{$\begin{array}{c}\text { операция } \\
\text { по поводу } \\
\text { прорыва } \\
\text { кисты в } \\
\text { желчные } \\
\text { протоки }\end{array}$} & \multirow{2}{*}{$\begin{array}{c}\text { отказ от } \\
\text { химиотерапии в } \\
\text { послеоперационном } \\
\text { периоде }\end{array}$} \\
\hline & & a6c. & $\%$ & & & \\
\hline $\begin{array}{l}\text { Echinococcus } \\
\text { veterinorum }\end{array}$ & 117 & 7 & 5,9 & 4 & 2 & 1 \\
\hline Echinococcus hominis & 97 & 9 & 9,3 & - & 1 & 8 \\
\hline $\begin{array}{l}\text { Echinococcus hominis } \\
+ \text { echinococcus } \\
\text { veterinorum }\end{array}$ & 8 & 1 & 12,5 & - & - & 1 \\
\hline $\begin{array}{l}\text { Echinococcus } \\
\text { acephalocystis }\end{array}$ & 14 & - & - & - & - & - \\
\hline Всего & 236 & 17 & 7,2 & 4 & 3 & 10 \\
\hline
\end{tabular}

В профилактике рецидива эхинококкоза важен правильный выбор способа операции в зависимости от характера осложнения и вида поражения органов паразитарным процессом.

Выводы: 1. Анализ морфологических характеристик эхинококковых кист печени по- казал, что чаще всего встречались echinococcus veterinorum (y 150 (49,2 \%) больных), echinococcus hominis (y 109 (35,7 \%) больных), echinococcus acephalocysticus (у 35 (11,5\%) больных) и ассоциация echinococcus hominis + echinococcus veterinorum (y 11 (3,6\%) пациентов). 
Огляди літератури, оригінальні дослідження, погляд на проблему

2. Результаты хирургического лечения эхинококковых кист печени во многом зависят от дифференцированного подхода к выбору адекватного способа оперативного лечения, учета морфологической формы эхинококковой кисты и соблюдения принципов апаразитарности и антипаразитарности.

\section{ЛИТЕРАТУРА}

1. Альперович Б.И. Хирургические вмешательства при эхинококкозе и пути профилактики рецидивов / Б. И. Альперович, Н. В. Мерзликин, В. Н. Сало // Анналы хирургической гепатологии. - 2005. - Т. 10, № 2. - С. 98.

2. Современный подход к хирургическому лечению эхинококкоза печени / Ш. И. Каримов, Н. Ф. Кротов, 3. И. Муртазаев, А. Э. Расулов // Хирургия Узбекистана. - 2007. - № 4. - С. 19-22.

3. Химиотерапевтическая профилактика рецидивных и резидуальных форм эхинококкоза брюшной полости/Ф.Г.Назыров, Х. А. Акилов, А. В.Девятов, У.М. Махмудов // Хирургия Узбекистана. - 2001. - № 3. - С. 78.

4. Химиотерапия и проблемы рецидивного эхинококкоза печени / Ф. Г. Назыров, А. В. Девятов, М. М. Акбаров [и др.] // Анналы хирургической гепатологии. 2011. - Т. 16, № 4. - С. 19-24.

5. Диагностика и хирургическое лечение эхинококкоза у детей / А. М. Шамсиев, А. Х. Одилов,
3. В отдаленном периоде рецидив эхинококкоза наблюдали у 2,9 \% больных, оперированных по поводу осложненного эхинококкоза, а также у 4,2 \% больных, отказавшихся от химиотерапии в послеоперационном периоде.
Д. О. Атакулов [и др.] // Детская хирургия. - 1999. № 5. - С. 17-20.

6. Современные аспекты морфологии, диагностика рецидивного эхинококкоза печени / А. М. Шамсиев, 3. Б. Курбаниязов, К. Э. Рахманов, С. С. Давлатов // Проблемы биологии и медицины. - 2015. № 3 (84). - С. 191-196.

7. Шамсиев Ж. А. Пути улучшения результатов хирургического лечения и профилактики рецидивов эхинококкоза у детей : дисс. на соиск. ученой степени док. мед. наук / Ж. А. Шамсиев.

8. Observations on clinical efficacy of albendazole emulsion in 264 cases of hepatic cystic echinococcosis / J. Chai, J. Wei, S. Deyu [et al.] // Parasitol Int. - 2004 № 53 (1). - P. 3-10.

9. Albendazole versus placebo in treatment of echinococcosis / M. Keshmiri, H. Baharvahdat, S. H. Fattahi [et al.] // Trans R.Soc.Trop.Med.Hyg. - 2001. - Vol. 95, № 2. - P. 190-194.

\section{IMPROVING THE DIAGNOSIS AND TREATMENT OF VARIOUS MORPHOLOGICAL FORMS OF LIVER ECHINOCOCCOSIS}

\section{A. M. Shamsiev, Sh. A. Yusupov, Z. B. Kurbaniyazov, K. E. Rakhmanov Samarkand State Medical Institute, Uzbekistan Republic}

SUMMARY. The Objective of the work was to find ways to optimize the diagnostic and therapeutic measures in patients with hepatic echinococcosis in view of the morphological forms of parasitic cysts. The study is based on a study of the results of the application of different options for surgical interventions in the treatment of 305 patients with liver echinococcosis. All patients in the last 10 years (2005-2015) have been treated in the II Clinic of Samarkand State Medical Institute.

Postoperative complications were observed in 35 (11.5\%) patients, postoperative mortality was noted in 1 (0.3\%) cases. Echinococcosis Relapse occurred in 17 of 236 patients observed in the long term (77.4\%).

The results of surgical treatment of hydatid cysts of the liver are largely dependent on a differentiated approach to the choice of an adequate method of surgical treatment, taking into account morphological form cyst and the principles aparazitarnosti and anti-parasitic.

In the late period of echinococcosis recurrence was observed in $2.9 \%$ of patients operated on for complicated echinococcosis, and in $4.2 \%$ of patients who refused chemotherapy postoperatively.

KEY WORDS: echinococcosis of the liver, morphological forms, diagnosis, treatment. 